\title{
Study on the Influence of the Sizes of Microbubble on Water Treatment
}

\author{
Zhekun $\mathrm{Li}^{1, \mathrm{a}^{*}}$, Wenli Shi ${ }^{1, \mathrm{~b}}$, Wei He ${ }^{1, \mathrm{c}}$, Yujin Fan ${ }^{1, \mathrm{~d}}$ and Chaojun Song ${ }^{2, \mathrm{e}}$ \\ ${ }^{1}$ School of Mechanical and Electrical Engineering, Kunming University of Science and Technology, \\ Kunming 650500, China \\ ${ }^{2}$ Yunnan Open University, Kunming 650093, China \\ azhekunlikust@sina.com, bswl_ie@163.com, hw_big@126.com, dfanyujinkmust@163.com, \\ e1187263598@qq.com
}

Keywords: microbubble; water treatment; air flotation method; influence factors

Abstract. In this paper, the development history and current situation of air floatation, the advantages and types of air flotation method, and the effects of flotation method on water treatment are expounded. The influence of the sizes of microbubble that is an important factor to the flotation effect on water treatment is studied. According to the actual situations, the sizes of the microbubble should be chosen. This paper has an important guiding role for the correct application of air flotation method on water treatment.

\section{Introduction}

In the water treatment, in order to separate the solid impurities from water, the sedimentation method is often used. However, the density of the object restrains the method, if the impurities have a similar density as water, the sedimentation separation method will not obtain the treatment effect. By the experience of mineral separation, the solid particles can be separated by flotation. Due to the adhesion of bubbles and impurities, the impurities that will have a smaller density than the water will be floated up and removed from the surface of the water. The air flotation method, compared with sedimentation method, has following advantages [1]: (1) a greater surface load, compact structure, small occupation area; (2) easily capturing tiny and light particles, the removal rate of small flocs can reach $80 \%-90 \%$, the removal rate of algae can reach $50 \%-80 \%$; (3) saving coagulant, or usually without any coagulant; (4) separation process can be controlled flexibly, adaptability is strong for water fluctuation interference; (5) decolorization, aeration and reducing COD. But what kind of bubbles in the water treatment is favorable, it is the research focus of this paper.

\section{History and Development of Air Flotation}

The air flotation method was first proposed by the German William Henie in 1860. At first the technology was mainly used in mineral separation. In the view of the theory of flotation, the researches for the early stage were mainly focused on the improvement of the flotation process. In 1905, an American put forward the pressurized dissolved air technology, in 1907, H. I. Norris invented the jet dissolved air flotation technology [2], in $20^{\text {th }}$ Century, in the 60s partial reflux pressurized dissolved air flotation (DAF) was appeared [3]. In the late of 70s, the dynamics and thermodynamics of air flotation was studied, because of the restriction of various factors at that time, the results were not great. In the late of $20^{\text {th }}$ Century, with the development of TS, TJ and other high performance aeration devices, the air flotation technology was used into many industrial fields and water treatment in many countries, such as Britain, France, Germany, Japan, Russia, Sweden, and so on.

A British water treatment company in 1975 first introduced a flotation device in the Aberden filtration station. In the next 5 years, more air flotation systems for the purification of water were established. A Business Company in France (Degremont) established the world largest air floating water treatment factory, the daily treating is 50,000 cubic meters of water. German, through the comparing study of the experiments, found that using air purification had better effect and higher efficiency than traditional sedimentation method. e Russian contributed much theoretical researches to 
the air flotation, but many of them were the experiments and less used in the actual productions. Japanese, in equipment and technology, had more extensive research and accessed a number of patents. Finlander used air flotation to clean water, from 1965 to 1992, 36 water treatment factories for drinking water were established, the total daily water purification capacity was $263,552 \mathrm{~m}^{3}$ and could supply for about one million people that was $20 \%$ of total population of Finland [4]. Swedish, as Finlander, had more than 100 large and small air flotation water treatment factories.

In China the use of air flotation technology started late, in the late of 1960s, dissolved air flotation devices were used in the salt making industry and oil wastewater treatment, due to technical problems, they were unrewarding [4]. In the design and test process of the wastewater treatment plant of Suzhou Cotton Textile Factory in 1975, the researchers found that the light weight particles in the printing and dyeing wastewater could be quickly gathered. Therefore, the air flotation method was used in the field test and was the first successful case in 1979. From 1977, partial reflux type pressure dissolved air floatation became the main direction of research of the National Urban Construction Bureau [1]. The air flotation technology that used in the daily water purification began in 1978 in the East Lake Wuhan waterworks. The purified water turbidity reached 2TNU and became the first successful case of air flotation to clean water. In this demonstration effect, more plants such as in Suzhou, Hebei, Shandong began to try to use air floatation to clean water. In 2007, Tianjin Jianyuan water plant had 500,000 tons daily capacity and was the rare largest water plant in China.

\section{Classifications of Air Flotation Methods}

Air floatation methods can be divided into four types according to the principle of generating air bubbles:

(1) Distributing air flotation. This method, generally by mechanical means, uses shearing force to make the air mixed within water become bubbles. For examples, a pump with suction pipe inhales air, a jet air, diffusion plates aerate air, an impeller shears air for flotation, etc.. This method has advantages of energy saving, simple layout, but has disadvantages of the bubble diameters not fine and the disturbance to the flocs in the water.

(2) Electrolysis air flotation. It is the electrolysis coagulation flotation. By the electrodes with the direct current, the cathode can produce microbubbles and have electrolytic coagulation effect. Although it is effective, the disadvantage is high cost of electrical power and the adverse effect of electrode passivation.

(3) The biological and chemical method. Biological air flotation process uses a biological role or adds flocculant into the water to produce microbubble. The effect of this method is not stable and limited by many conditions, so the application is less.

(4) Dissolving air flotation. It can be divided into the vacuum air flotation and the pressure dissolving air flotation. The principle of vacuum air floatation can make the air in the water release and generate microbubbles in the normal condition. Pressure dissolving air flotation method presses the air in the water, and then the pressure is immediately decreased, so that the air releases and generates microbubbles. The two methods are similar, but each has different advantages and disadvantages. Vacuum air flotation is less energy consumption, and the water body is stable, but in the actual standard pressure, the content of air dissolved in water is less, it is not easy to meet the needs of the floatation operation. Moreover, because within the equipment the pressure requirements are below the outside air pressure, the obturation performance needs to be good and the maintenance cost is high, so it is rarely used now. Pressure dissolving air flotation method adopts artificial pressure and then suddenly is decompressed, the air content in the water relates on how much the pressure is. The bubble diameter is smaller, the density is uniform, and floatation is stability. It is suitable for the separation of loose and small impurities. But it needs to dissolve air into the water by a high air pressure.

In comparison, the two methods of distributing air flotation and electrolysis air flotation are widely applied. If the generating microbubbles can be solved better, the distributing air flotation can be applied more widely. 


\section{Effects of the Sizes of Microbubble on Water Treatment}

In the process of flotation, by various reactions, flocculation and microbubbles can float to the surface of the water. The floatation process of the microbubbles in the water can be expressed by the Newton's formula:

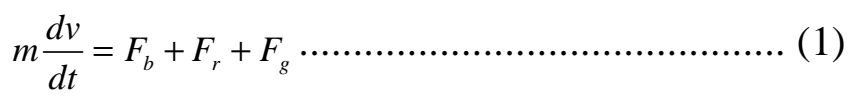

$F_{b}$ - Buoyancy of an object, N; $F_{r}$ - Resistance of water, N; Fg - Floating object gravity, N.

$F_{b}=\rho_{w} g V, F_{r}=\frac{C A \rho_{w} v_{f}^{2}}{2}$ and $F_{g}=\rho_{f} g V, \rho_{f}$ is Floating object density, $\mathrm{kg} / \mathrm{m}^{3} ; \rho_{w}$ is Density of water, $\mathrm{kg} / \mathrm{m}^{3}$; $\mathrm{C}$ is Resistance coefficient, dimensionless, 0.1-0.8; A is Projection area in the direction of the flow velocity of the water, $\mathrm{m}^{2} ; \mathrm{g}$ is Acceleration of gravity, $\mathrm{m} / \mathrm{s}^{2} ; \mathrm{V}$ is Floating volume, $\mathrm{m}^{3}$.

When the initial acceleration of the floating flocs is zero, the volume of the dirt is small and the shape is considered as a ball, the velocity formula of the dirt floating to the surface of water can be obtained by $\mathrm{C}=0.4$ :

$$
v_{f}=1.83 \sqrt{\frac{\rho_{w}-\rho_{f}}{\rho_{w}} g d}
$$

$\mathrm{d}$ is the diameter of the dirt, $\mathrm{m}$.

For the Reynolds number of the fluid, there are different forms. For examples, when the fluid is dominated by the laminar flow, the dirt floating velocity to the surface of water expressed by the Stokes is:

$$
v_{f}=\frac{g}{18 \mu}\left(\rho_{w}-\rho_{f}\right) d^{2}
$$

$\mu$ in the formula is dynamic viscosity coefficient.

When the fluid flows both laminar and turbulent, for this transition section the Allen formula is used:

$$
v_{f}=0.22\left(\frac{\rho_{w}-\rho_{f}}{\rho_{w}}\right)^{2 / 3} \frac{d}{v^{1 / 3}}
$$

$v$ in the formula is the kinematic viscosity coefficient.

From the Equations of 2, 3, 4 it can clearly know that the dirt with bubble floating velocity to the surface of the water is mainly decided by three factors: (1) the difference density of dirt with bubble to the water, (2) the fluid flow state: laminar or turbulent or intermediate process, (3) the diameter of the bubbles. Where, if the diameter of the bubble is small, one unit volume of dirt can adhere more bubbles, the density of dirt with bubble is smaller, floating speed will significantly improve. The faster the floating velocity is, the shorter the time of water separation is and the higher the separation efficiency is, too. That is one of the important advantages of the air flotation method compared with the traditional method of sedimentation.

The size of the bubble also has certain effect on adhesion and probability of collision between bubbles and dirt. The collision probability was first studied in mineral separation, from research of Yoon.R.H, et al. Some mineral (dirt) in the pulp is trapped by bubbles, the trapping probability empirical formula is:

$$
E=E_{c} E_{a}\left(1-E_{d}\right)
$$

Ec is the collision probability between microbubbles and dirt; Ea is the adhesion probability of microbubble and dirt; Ed is the probability of the detachment of microbubble and dirt; All are dimensionless.

Since the middle of last Century, some researchers had studied the probabilities of the impact of microbubbles and the probabilities of adhesion. Through theoretical analysis and experiments, a number of empirical formulas for adhesion and collision probability were presented. A famous one is by Yoon. R. H: 


$$
E_{a}=\operatorname{sech} 2\left(\frac{2 u_{b} A t_{i}}{d_{p}+d_{b}}\right)
$$

The values of $u_{b}$ and A can be determined by the following formulas:

$$
\begin{aligned}
& u_{b}=\frac{g d_{b}^{2} \rho_{l}}{18 u_{l}\left(1+0.15 \operatorname{Re}_{b s}^{0.687}\right)} \\
& A=\frac{3 d_{p}}{2 d_{b}}+O\left(\frac{d_{p}}{2 d_{b}}\right)^{2}
\end{aligned}
$$

It is clear that if the diameter of the dirt is certain and the diameter of the microbubble reduces, it can directly improve the collision probability of microbubbles and dirt. The greater the probability of collision is, the faster adhesion is, the flotation separation efficiency will be greatly improved. The direct relationship between the size of bubble and the flotation efficiency can be obtained by the dynamic theory of air flotation [5]:

$$
k=\frac{3\left[E_{a} d_{p}\left(1.5+4 R_{e}^{0.75} / 15\right) V_{g}\right]}{2 d_{b}^{3}}
$$

The $\mathrm{K}$ is a rate constant, which expresses adhesion speed of the microbubbles and contaminants. The Equation 12 is the relationship between the rate constant and the diameter of the microbubble, and the value of $\mathrm{K}$ increases greatly as the diameter of microbubbles decreases, the rate constant $\mathrm{K}$ is inversely proportional by the three order of the diameter of the bubble [6].

By reducing the size of the microbubble, it is an effective and stable way to improve the separation efficiency. But it must pay more attention to that [7] if the diameter of the microbubble is less than a certain small degree, it will have an adverse effect on the floating velocity. According to the experimental results of Kiuru [3], the size of the bubble is too small, the effect of air flotation water treatment is not too better.

\section{Conclusion}

Since the air flotation method was invented, its application scope has been expanded gradually, and it has special effect on water treatment. But in the process of using, the size of the bubble has important influence on the result of water treatment and the efficiency of the process. In this paper, within a reasonable range the size of the microbubble is smaller, it will be more beneficial to improve the adhesion and collision probability between the bubbles and the dirt, shorten the time and improve the efficiency of water treatment. This paper has an important reference for the correct application of air flotation method in water treatment.

\section{Acknowledgements}

This work was sponsored by China Natural Science Foundation Project (No. 51168020) and Yunnan Province Natural Science Foundation Project (No. 2013FZ024). We will thank here!

\section{References}

[1] Wang Jingchao, Ma Jun, Wang Jinghai. Application and Research Situation of Air Flotation Technology in Water Treatment, Industrial Water Treatment, 2004, 24 (7), pp 9-12.

[2] Kitchener, J. A. The Air Flotation Process: Past, Present, and Future-In Brief. Scientific Basic of Flotation, 1984, (1) , pp 3-51.

[3] Kiuru H. J. Development of Dissolved Air Flotation Technology from the First Generation to the Newest One. Wat. Sci. Tech., 2001, 43(8), pp 1-7. 
[4] J. Heinänen, P. Jokela, T. Ala-Peijari. Use of Dissolved Air Flotation in Potable Water Treatment in Finland. Wat.Sci.Tech.1995, 31 (3-4), pp 225-238.

[5] Dai Jinlong, Xie Guangyuan, Liu Shanshan, et al. Analysis of Factors Affecting the Size of Flotation Bubble. Coal Preparation Technology, 2007, 10 (5) , pp 7-9.

[6] A Barnea. Unified model for Predicting Flow Pattern Transitions for Whole Range of Pipe Inclinations. Int. J. Multiphase Flow, 1987, (13) , pp 1-12.

[7] Yang Yong, Wang Guangfeng, Liu Long. Analysis on Relationship between Diameter of Air-Bubble and Effect of Air-Floatation on Water Purification. Industrial Water and Wastewater. 2007, 8 (4), pp 18-19. 